\title{
ATLAS Discovery Potential of the Standard Model Higgs Boson
}

Christian Weiser University of Freiburg (on behalf of the ATLAS Collaboration)

$14^{\text {th }}$ Lomonosov Conference on Elementary Particle Physics Moscow, August 2009 


\section{Content}

- Introduction

- Higgs search channels in ATLAS

$-\mathrm{H} \rightarrow \gamma \gamma$

$-\mathrm{VBF} H \rightarrow \tau \tau$

$-H \rightarrow b b$

$-H \rightarrow Z Z$

$-H \rightarrow W W$

- Combined sensitivity

- Summary 


\section{What do we already know?}

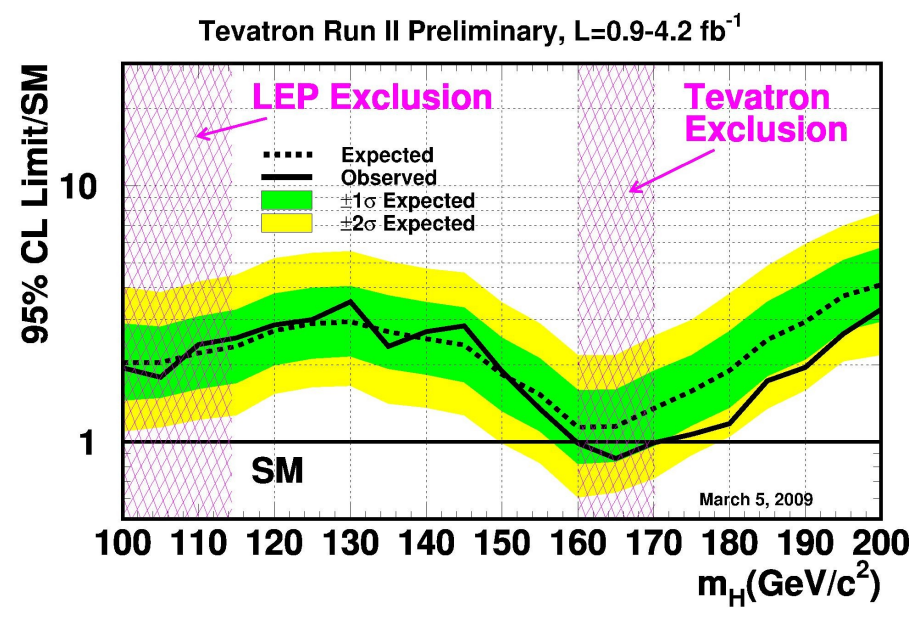

- Direct searches at LEP

$$
M_{H}>114.4 \mathrm{GeV} / \mathrm{C}^{2} \quad(95 \% \mathrm{CL})
$$

- Direct searches at the TEVATRON

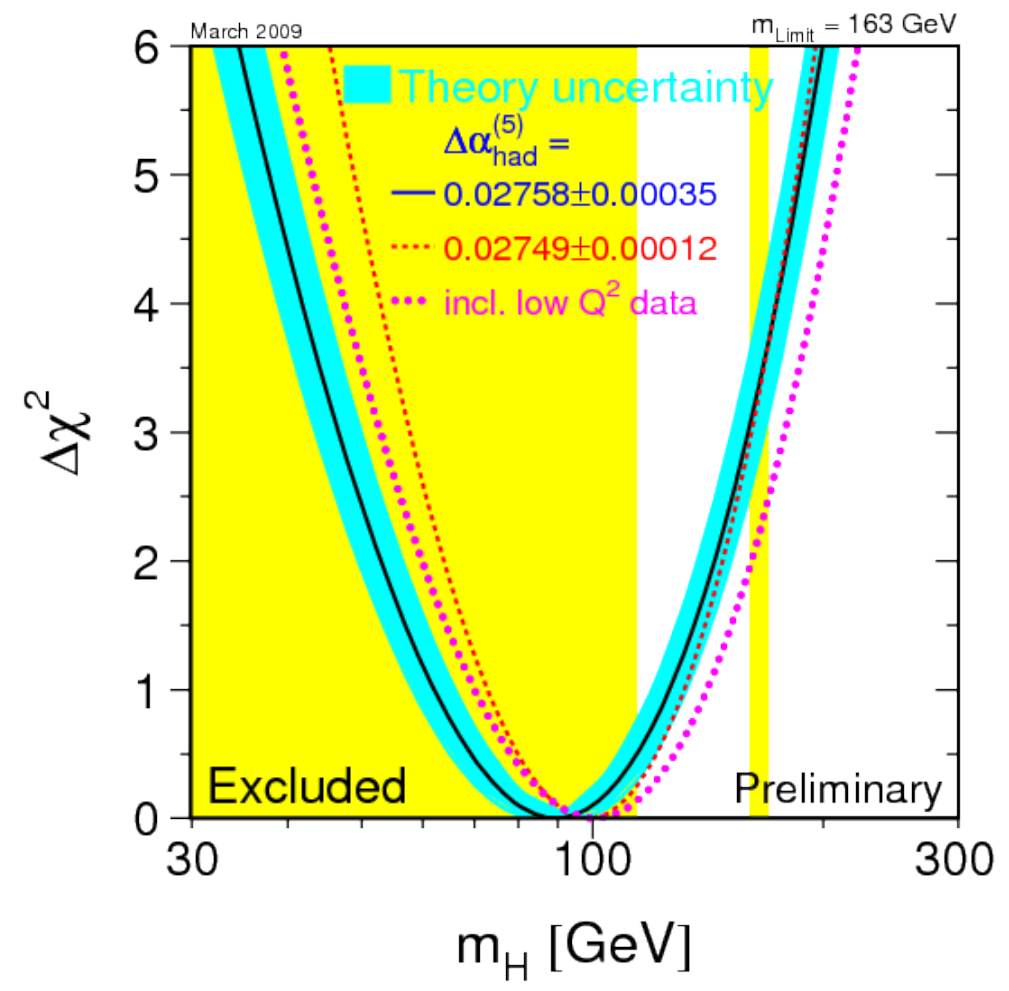

Exclude $160 \mathrm{GeV} / \mathrm{c}^{2}<M_{H}<170 \mathrm{GeV} / \mathrm{c}^{2}$ $(95 \% C L)$

- electroweak precision measurements $M_{H}<163 \mathrm{GeV} / \mathrm{c}^{2} \quad(95 \% \mathrm{CL})$ (191 GeV/c $\mathrm{c}^{2}$ incl. LEP Limit) 


\section{Higgs Boson Production}

\section{(i) Gluon-Fusion}

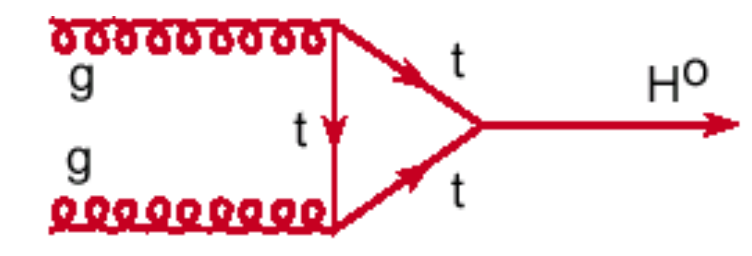

(ii) Vector Boson Fusion (VBF)

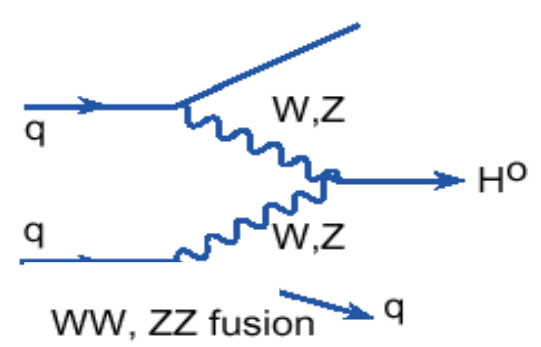

(iii) Associated Production $(W / Z, t+)$
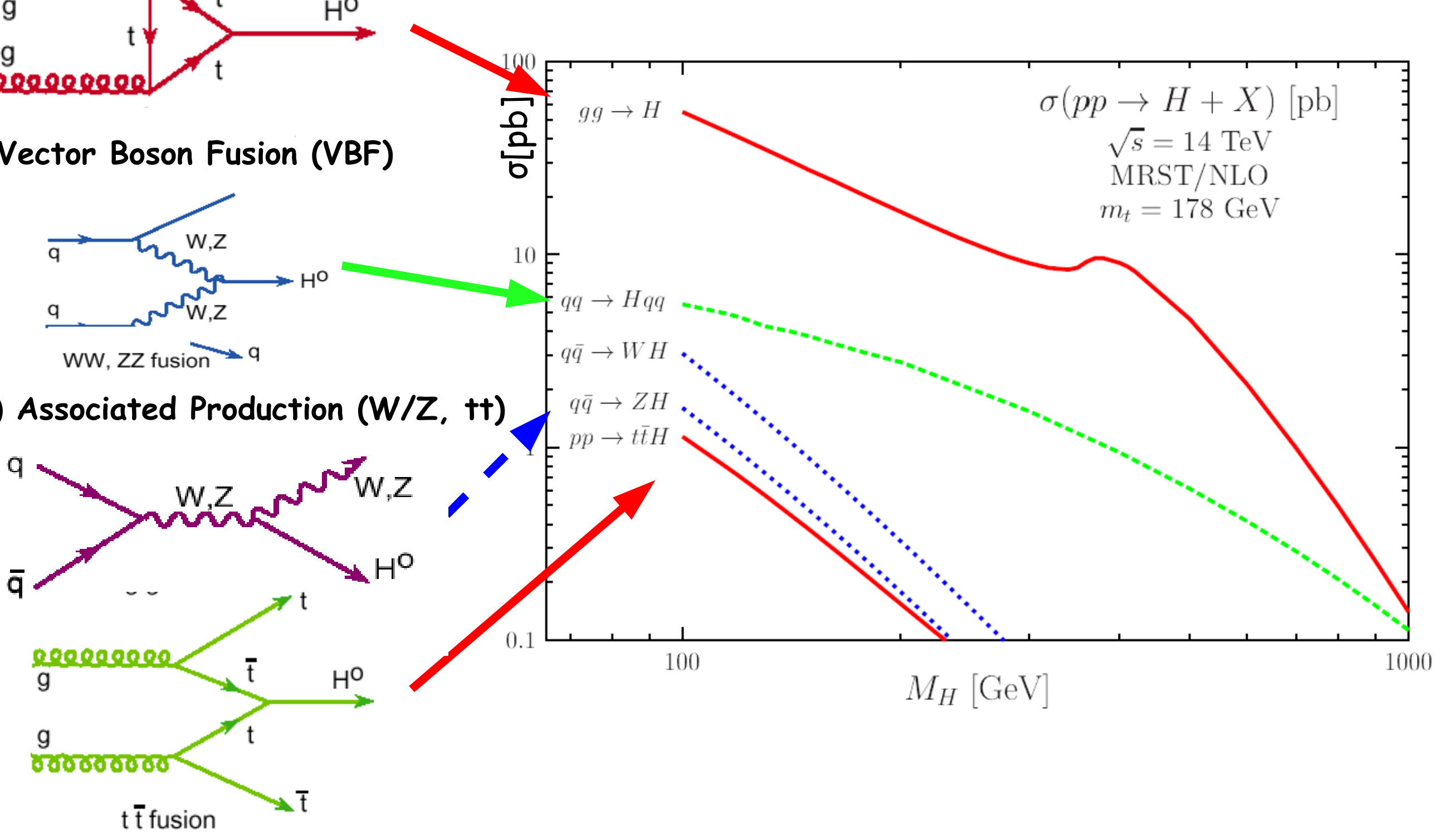


\section{Higgs Boson Decays}

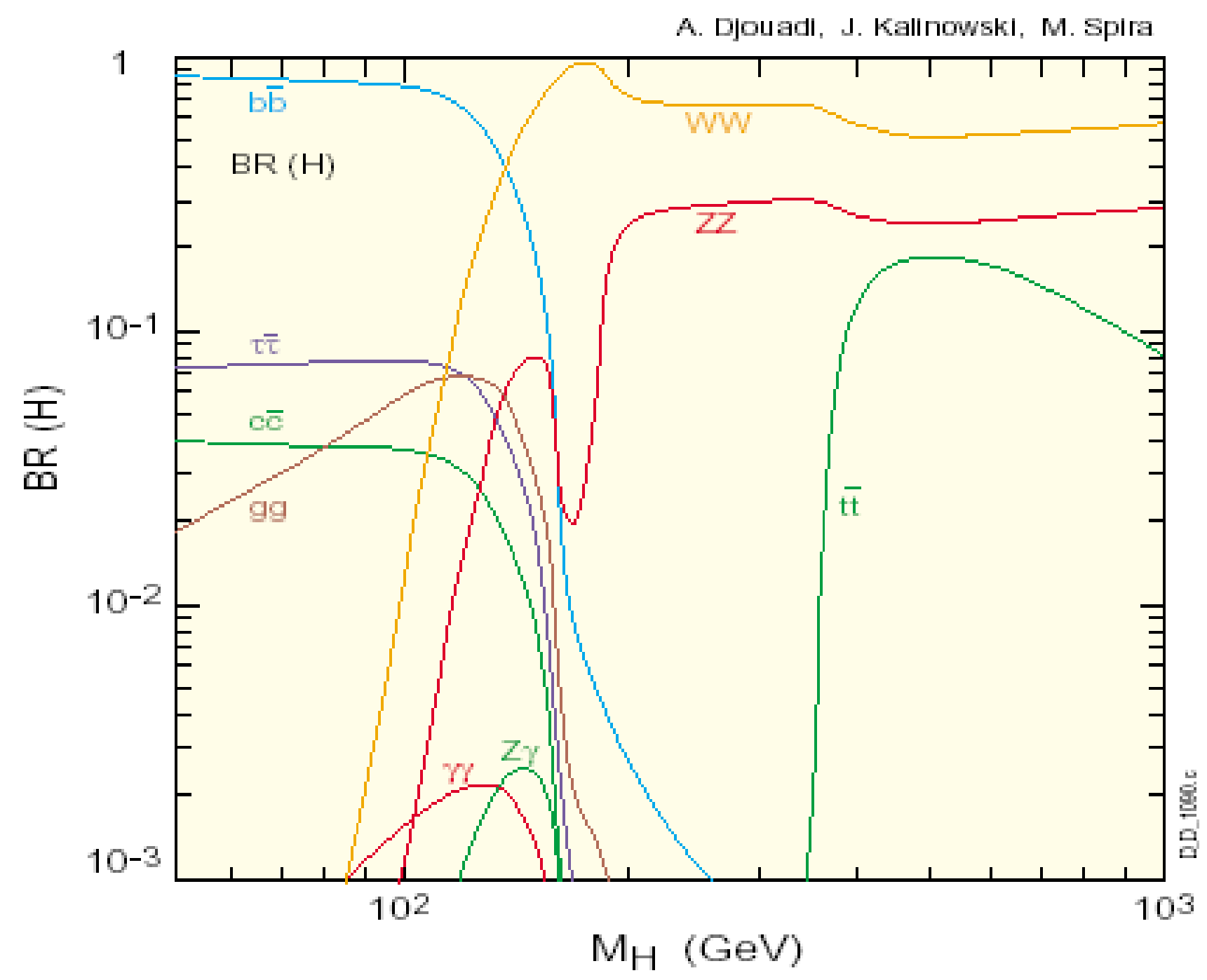

C.Weiser ATLAS SM Higgs

$14^{\text {th }}$ Lomonosov Conference, Moscow

$24 / 8 / 2009$ 


\section{Search Channels}

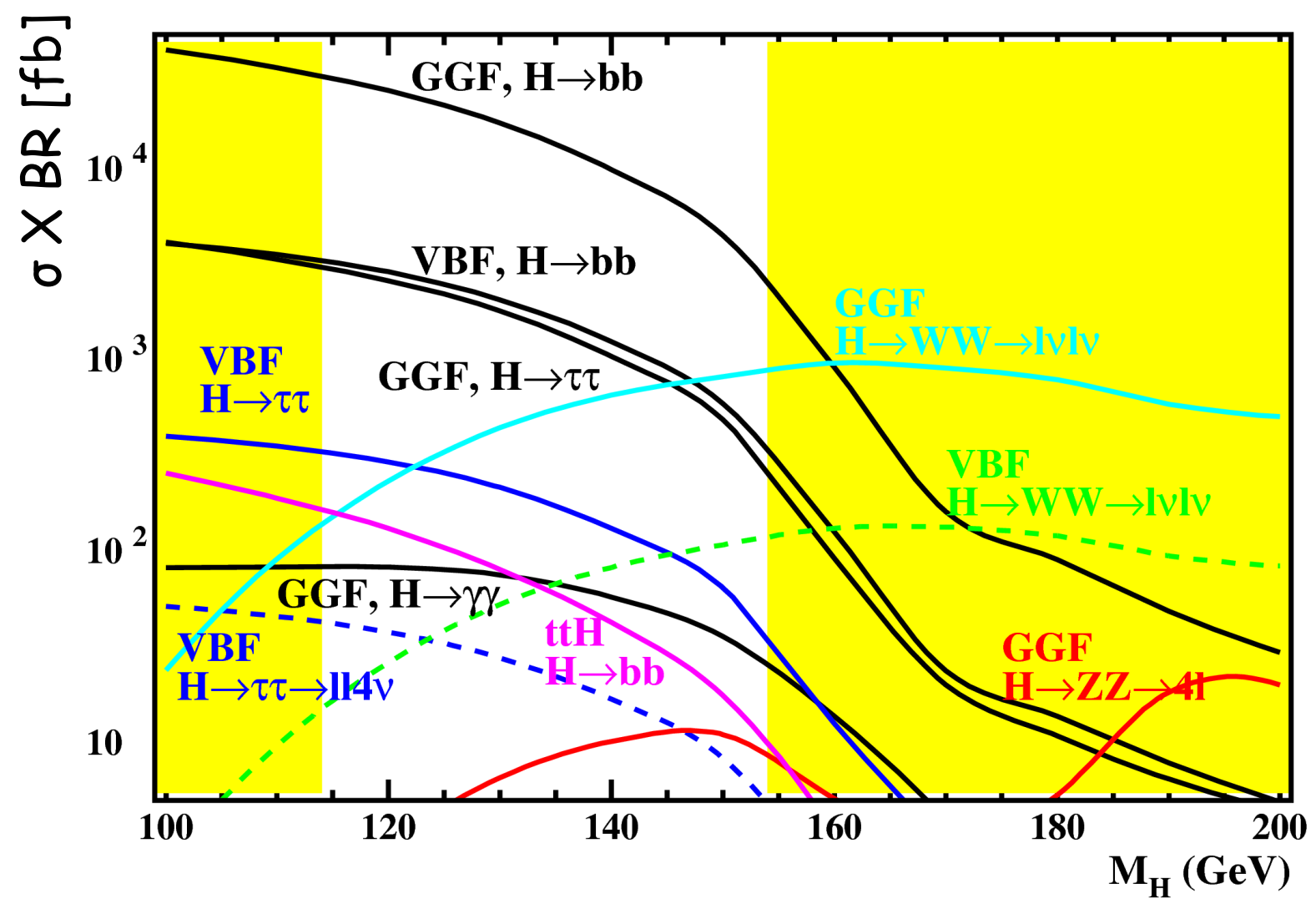

Main channels studied:

Low mass:

$-\mathrm{H} \rightarrow \mathrm{YY}$

$-q q H, H \rightarrow \tau \tau$

$-t+H, H \rightarrow b b$

$-W / Z H, H \rightarrow$ bb NEW!

Also medium ( $\&$ high) mass:

$-\mathrm{H} \rightarrow \mathrm{WW} \mathrm{W}^{(*)}$

$-H \rightarrow Z Z^{(*)}$

For low mass: Combination of large production cross section (gg-fusion) and large $B R(H->b b)$ does not work because of $Q C D$ background 


\section{Higgs Studies in ATLAS}

Studies presented here:

- Realistic (at least we believe ...) GEANT4 based detector description simulation

- Real reconstruction algorithms

- Trigger simulation

- Effects of additional minimum bias events (Pile-Up), misalignment etc. studied

- Improved generators (NLO etc.)

- Background estimation from data in many cases

For all details see CERN-OPEN-2008-020

Focus in this talk:

Discovery potential in the low and medium mass range $(\mathrm{mH} \leq 200 \mathrm{GeV}) @ \sqrt{s}=14 \mathrm{TeV}$

To be (re)studied:

Parameter determination (Mass, Spin, CP, Couplings, ....) Heavy Higgs bosons 


\section{$H \rightarrow \gamma \gamma$}

No direct coupling

tiny $B R(\approx \%$ )

$\sigma \times B R \approx 100 \mathrm{fb}$

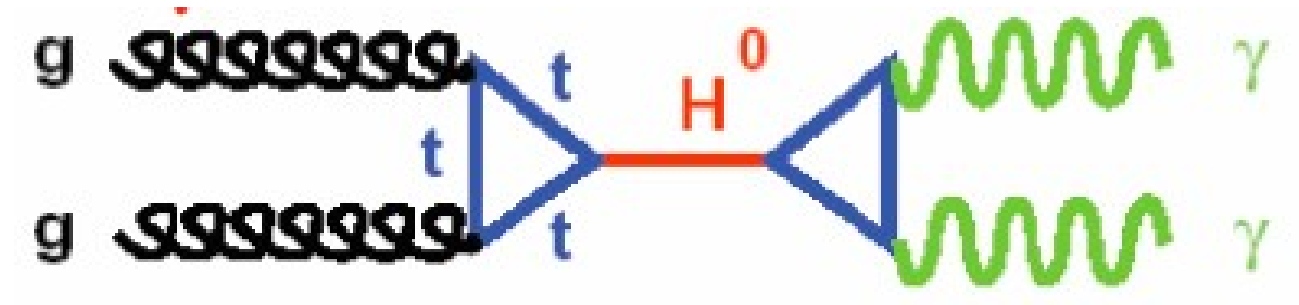

Backgrounds:

- Irreducible $\gamma \gamma(+X)$

$\left(\sigma \approx 30 \mathrm{pb}\right.$ for $\mathrm{p}_{\mathrm{T}, \mathrm{v}}>25 \mathrm{GeV},|n|<2.5$ $80 \mathrm{GeV}<m_{v v}<150 \mathrm{GeV}$ )
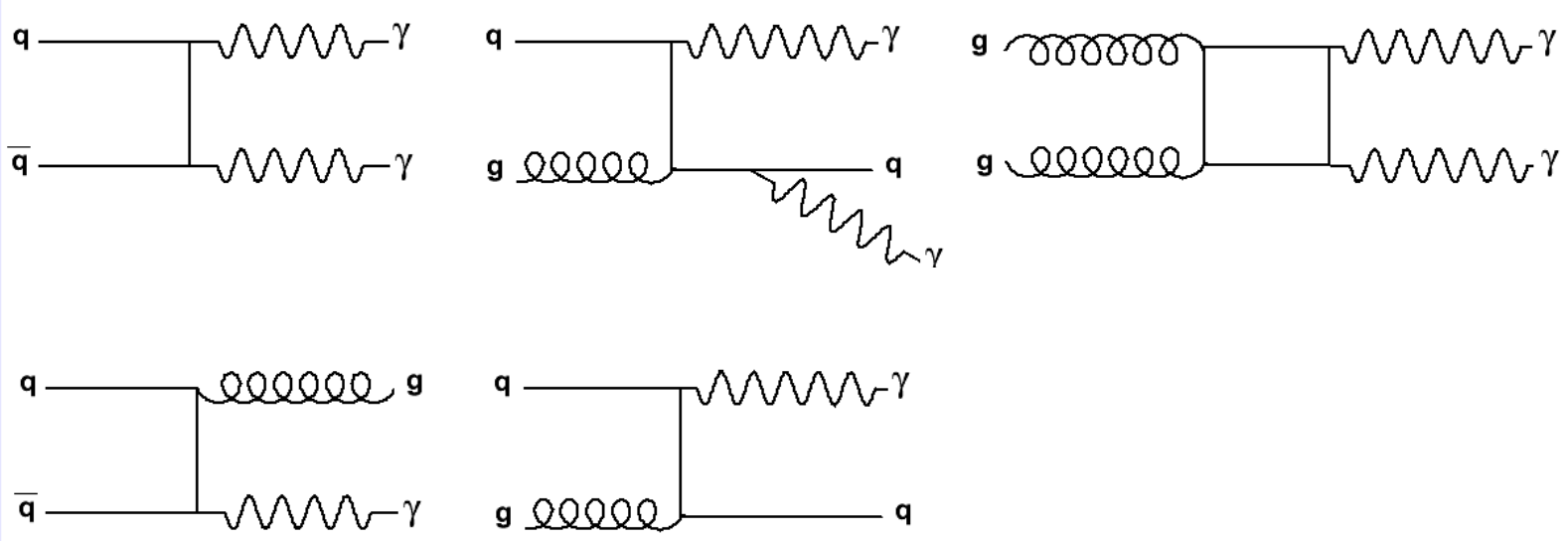

- Reducible $\gamma+$ jet, jet+jet $\left(\gamma \mathrm{j}: \sigma \approx 180 \mathrm{nb}\right.$ for $\mathrm{p}_{\mathrm{T}, \gamma}>25 \mathrm{GeV}$ $\mathrm{jj}: \sigma \approx 480 \mu \mathrm{b}$ for $\mathrm{p}_{\mathrm{T}}>25 \mathrm{GeV}$ )

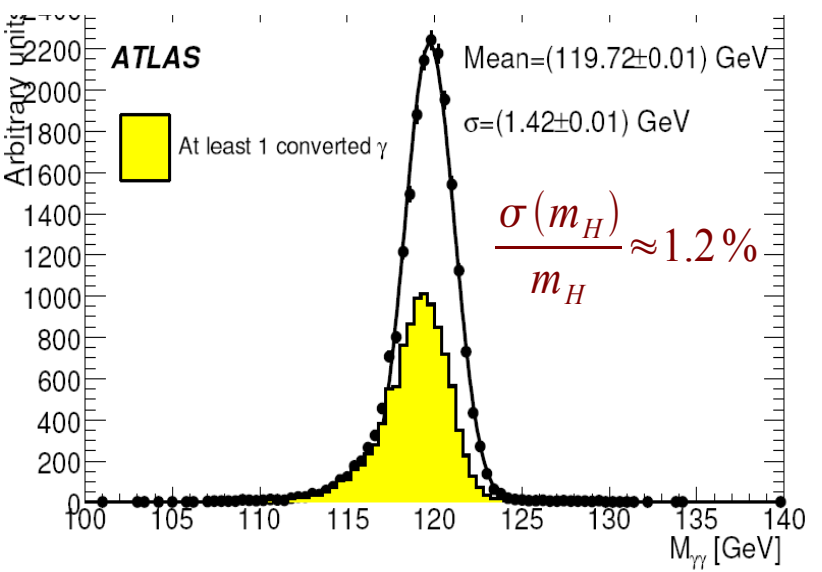

Need excellent

- Photon identification

- Photon-jet separation

- Mass resolution $\approx 50 \%$ of events:

at least 1 photon conversion

$\rightarrow$ reconstruct from $e^{+}, e^{-}$tracks in Inner Detector 


\section{$H \rightarrow \gamma \gamma$}

Inclusive (ask for photons only) analysis:

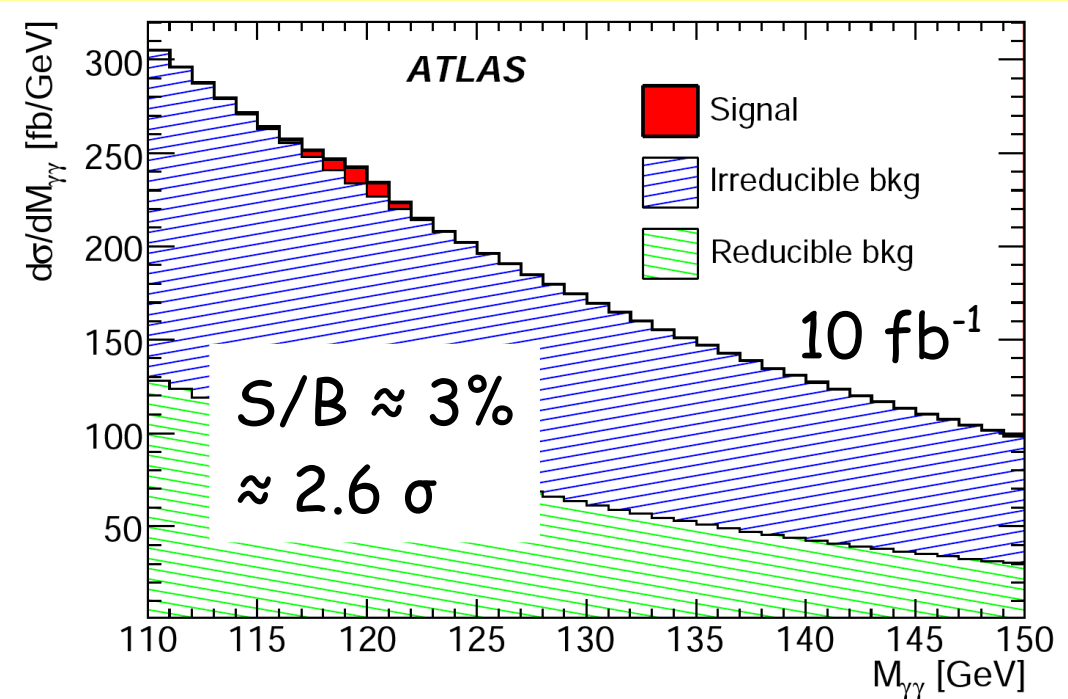

\section{Discovery Potential (counting)}

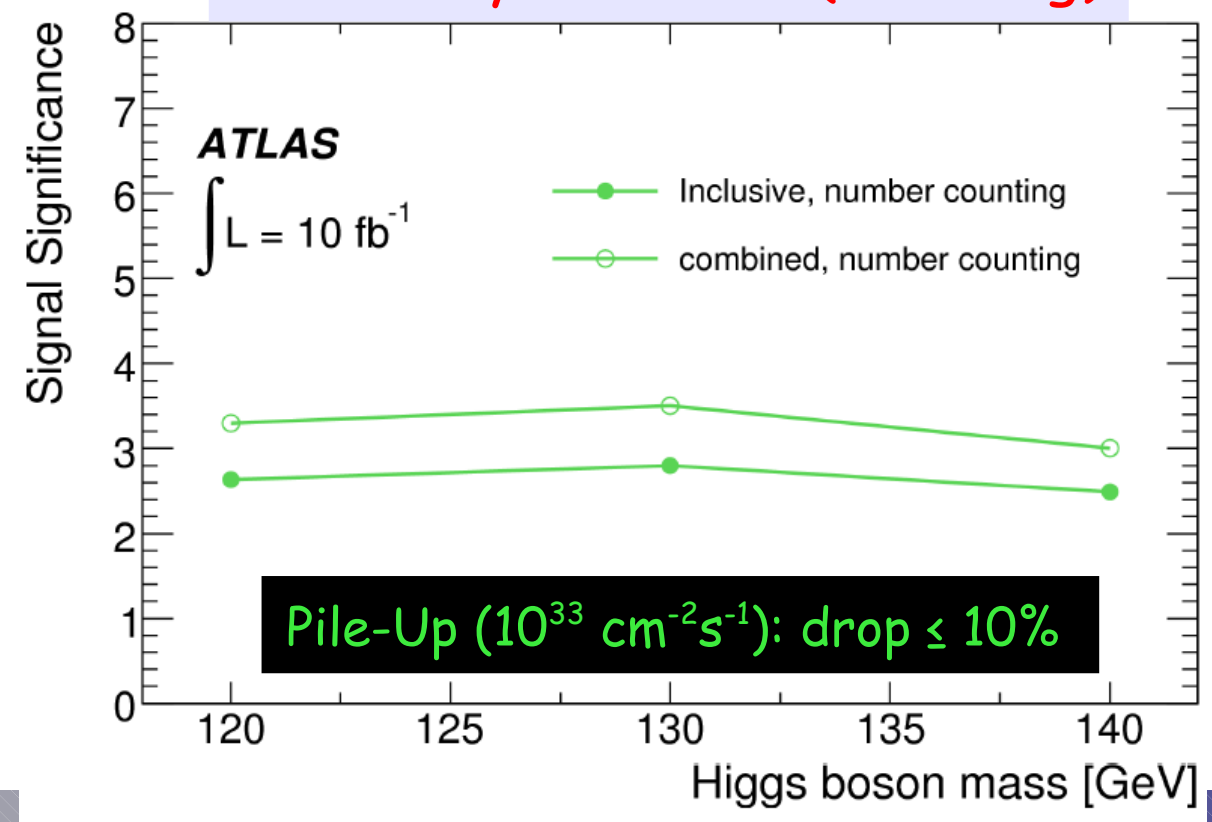

$\gamma \gamma+1,2$ jet analyses (enhance VBF): $10 \mathrm{fb}^{-1}$

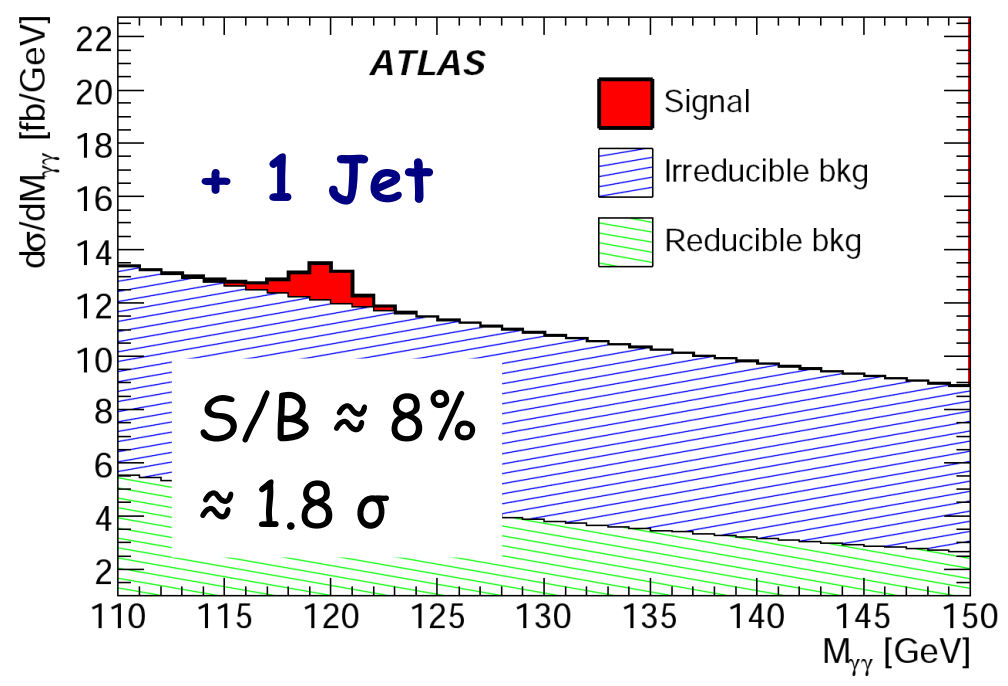

$10 \mathrm{fb}^{-1}$

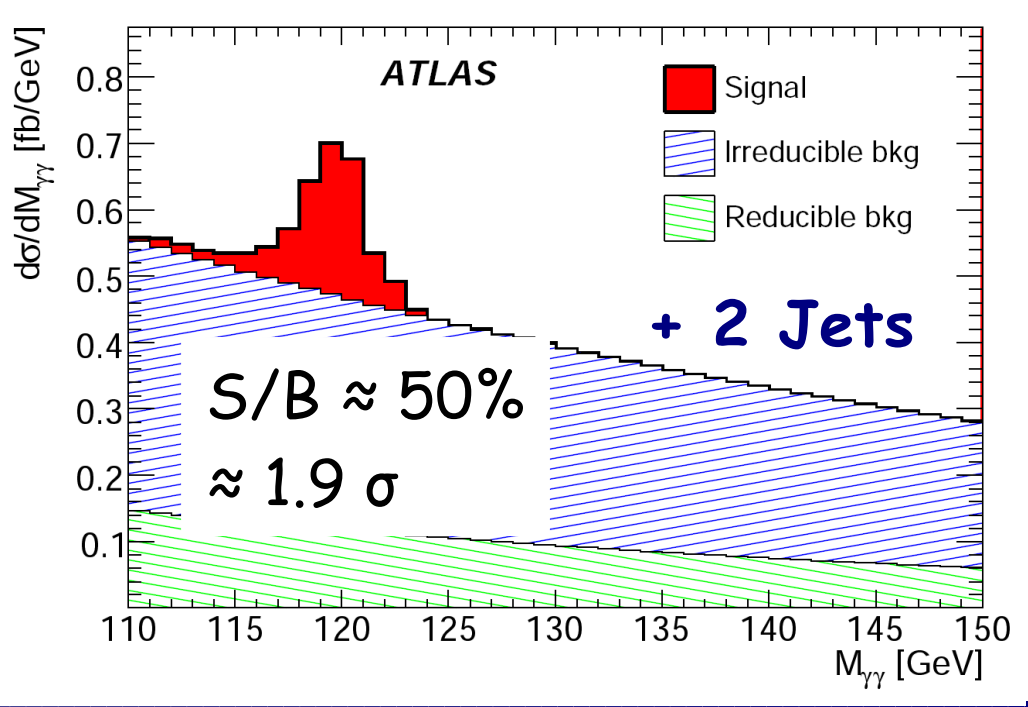




\section{Vector Boson Fusion $\mathrm{qqH}, \mathrm{H} \rightarrow \tau \tau$}

\section{Signature of Vector Boson Fusion (VBF)}

Production process:

- Two hard forward/backward "Tagging Jets" with large rapidity gap and high invariant mass

- Jet activity suppressed in central part of the detector $\rightarrow$ Central Jet Veto

- Large discrepancies between generators

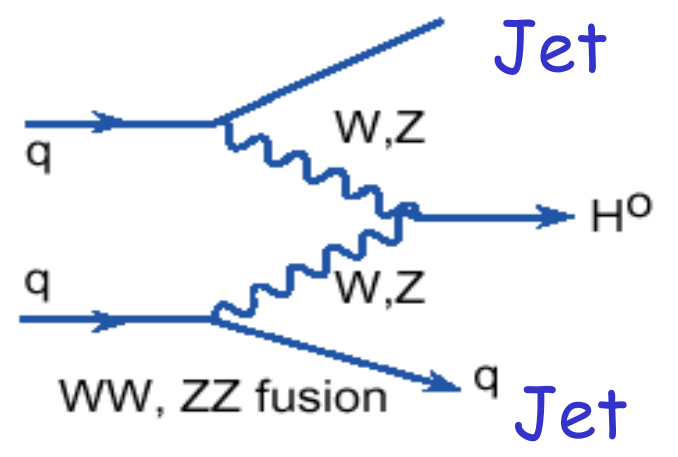

Main Backgrounds:

- $Z(\rightarrow \tau \tau)+$ jets

- $W(\rightarrow \tau v)+$ jets

$\cdot+\dagger$

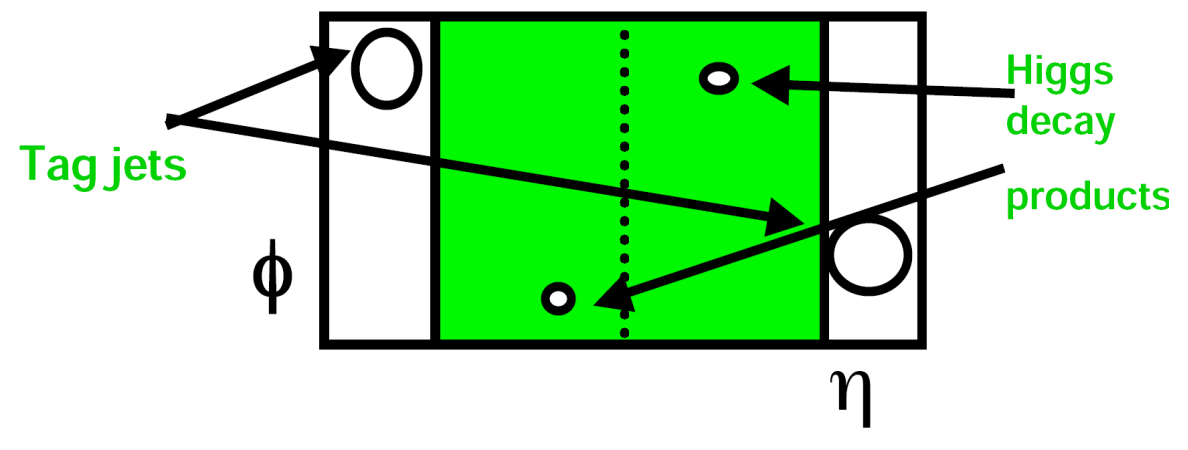

Rapidity Gap

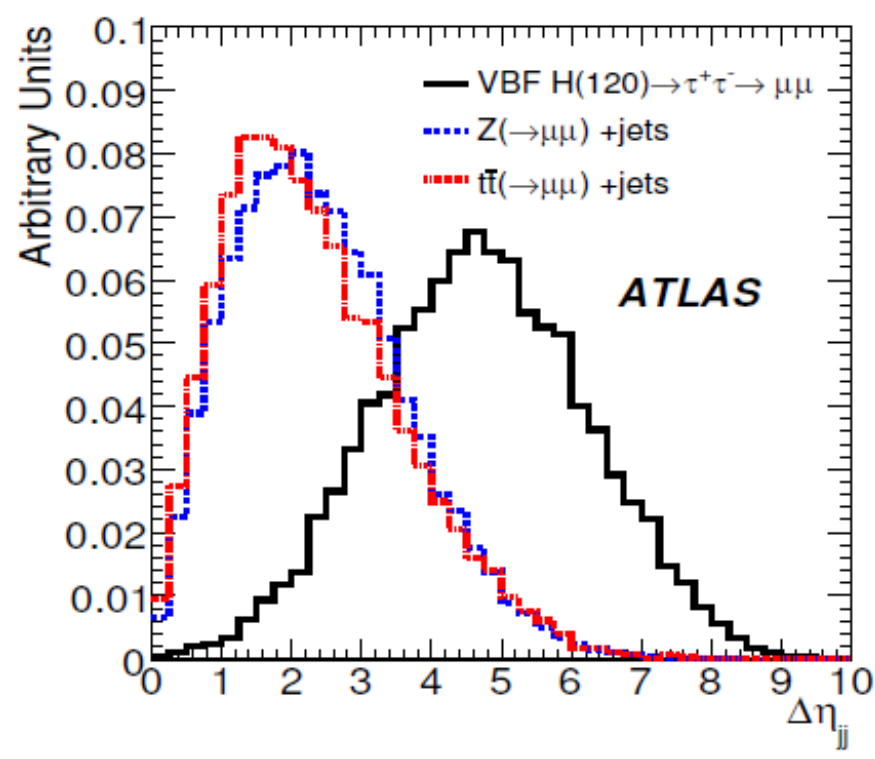

Central Jet Veto

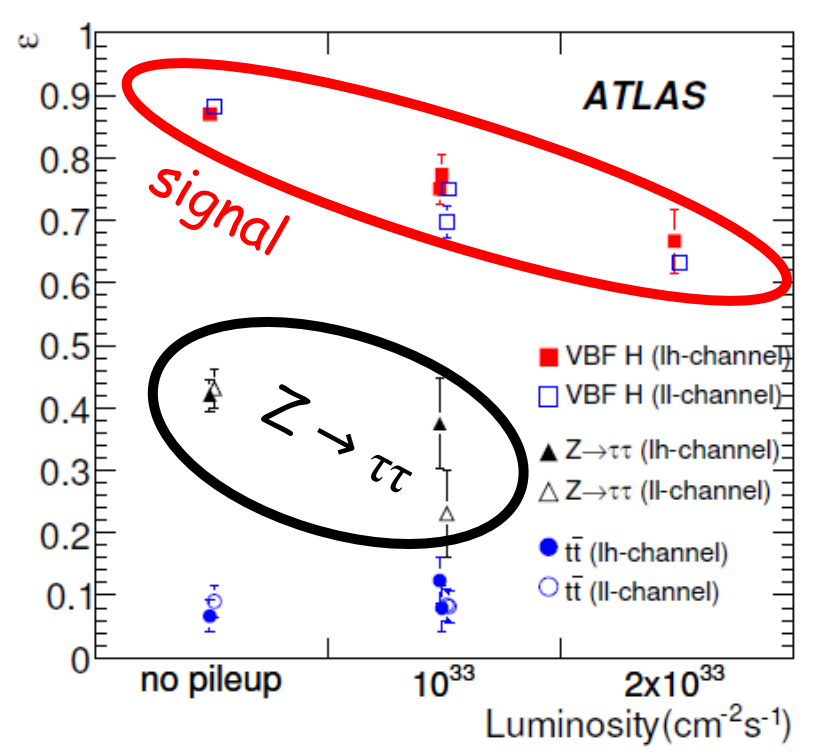




\section{Vector Boson Fusion $\mathrm{qqH}, \mathrm{H} \rightarrow \tau \tau$}

T decay channels:

- Lepton-lepton (II)

- Lepton-hadron (Ih)

- [Hadron-hadron (hh)] $\vec{p}_{2}^{v i s}$

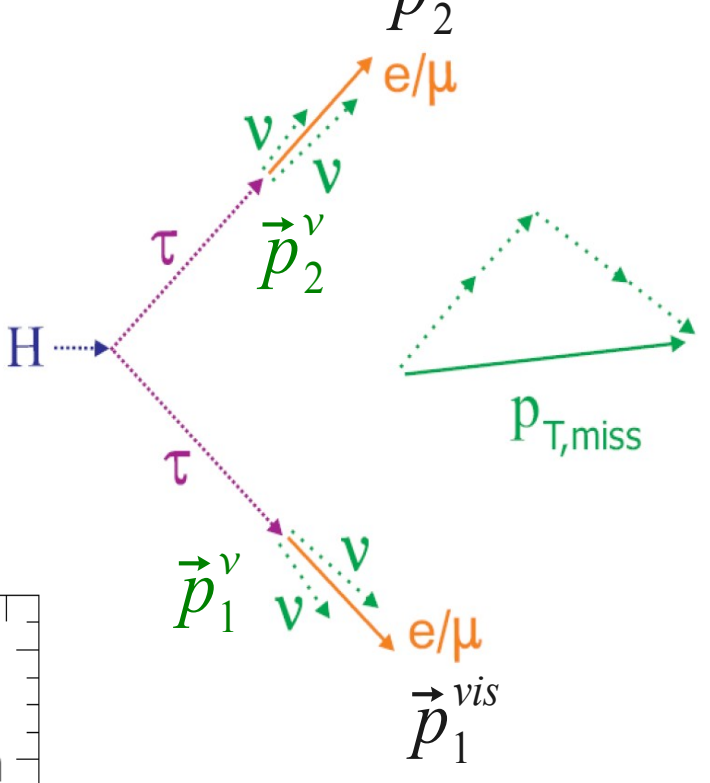

Dominating

Background

$Z \rightarrow \tau \tau$ :

Estimate on data from $Z \rightarrow \mu \mu$ events with $\mu \rightarrow \tau$ transformation
Invariant mass computation: ( $>1$ neutrino in final state) "Collinear Approximation" (Crucial: MET resolution)

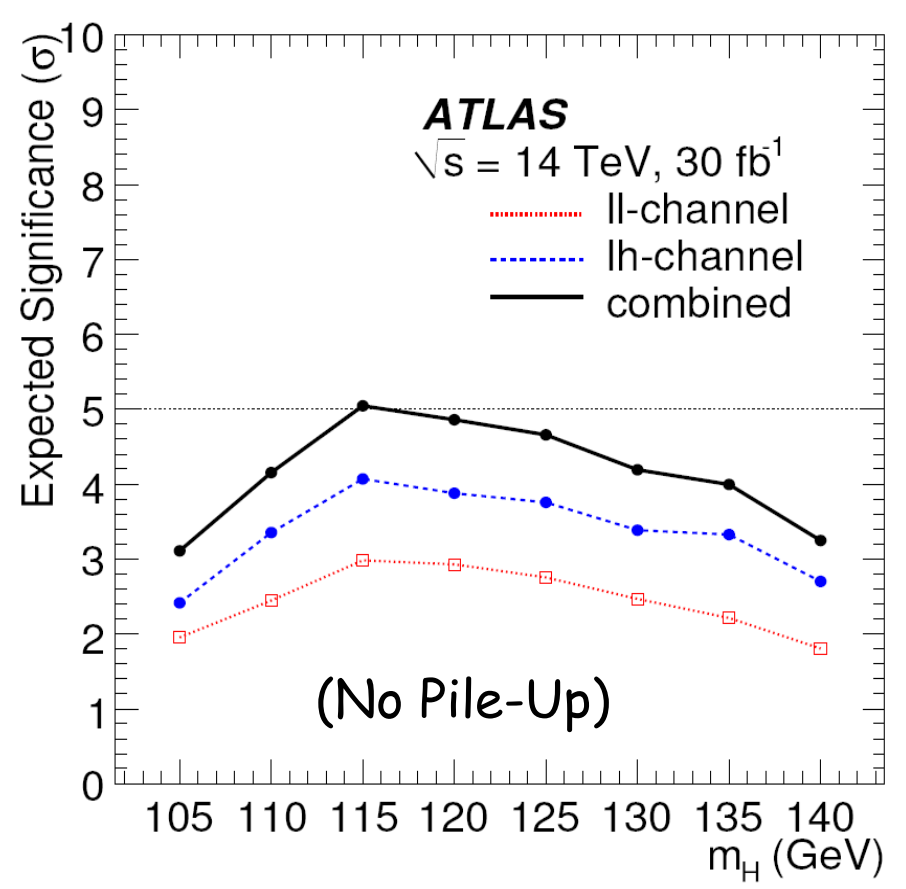

hadron-hadron final state: No reliable estimate of $Q C D$ background without data $\rightarrow$ No expected significance quoted 


\section{$H \rightarrow b b$}

t+H a promising search channel some years ago:
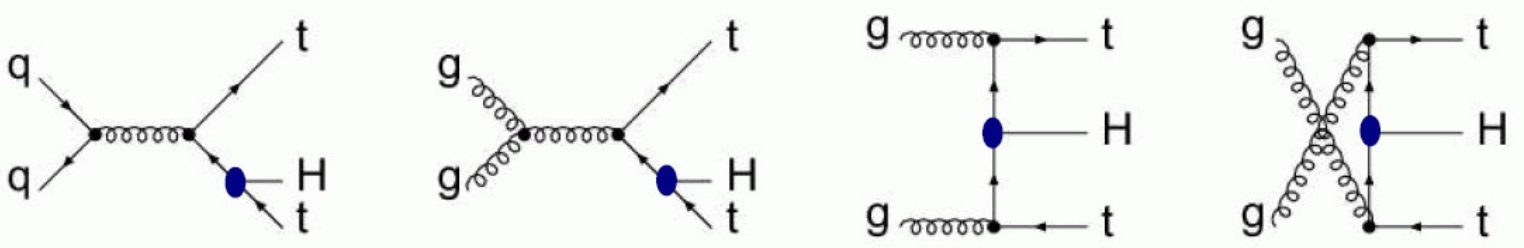

Access to top-Higgs Yukawa coupling!

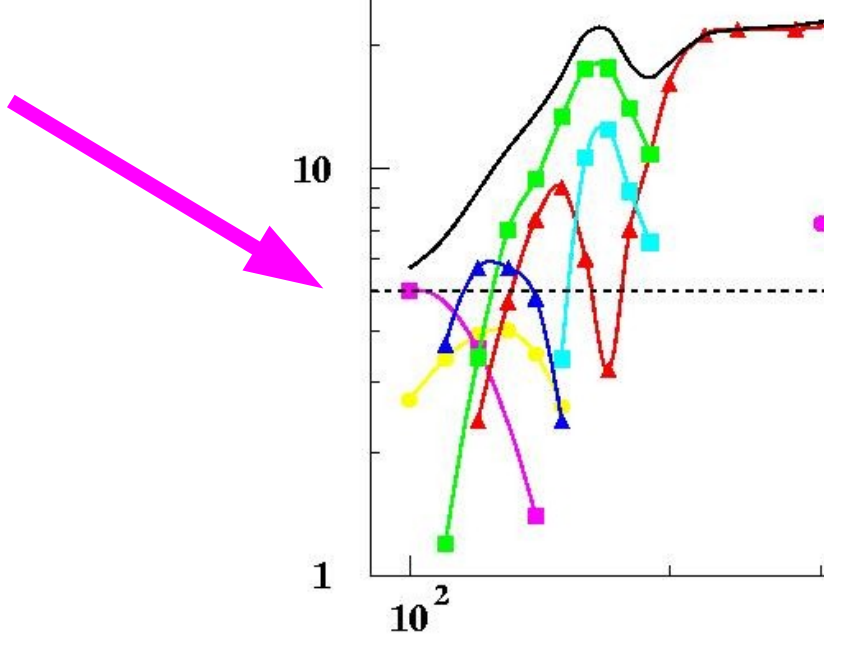

Complex final state:

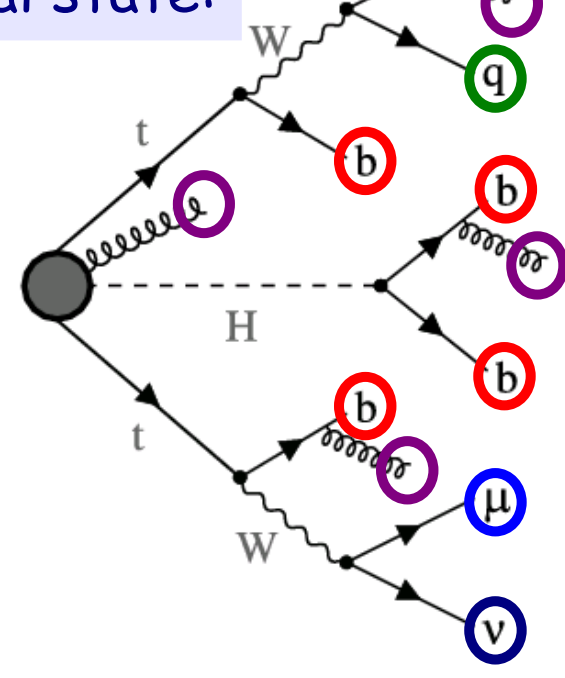

Main Backgrounds: ttbb, ttjj
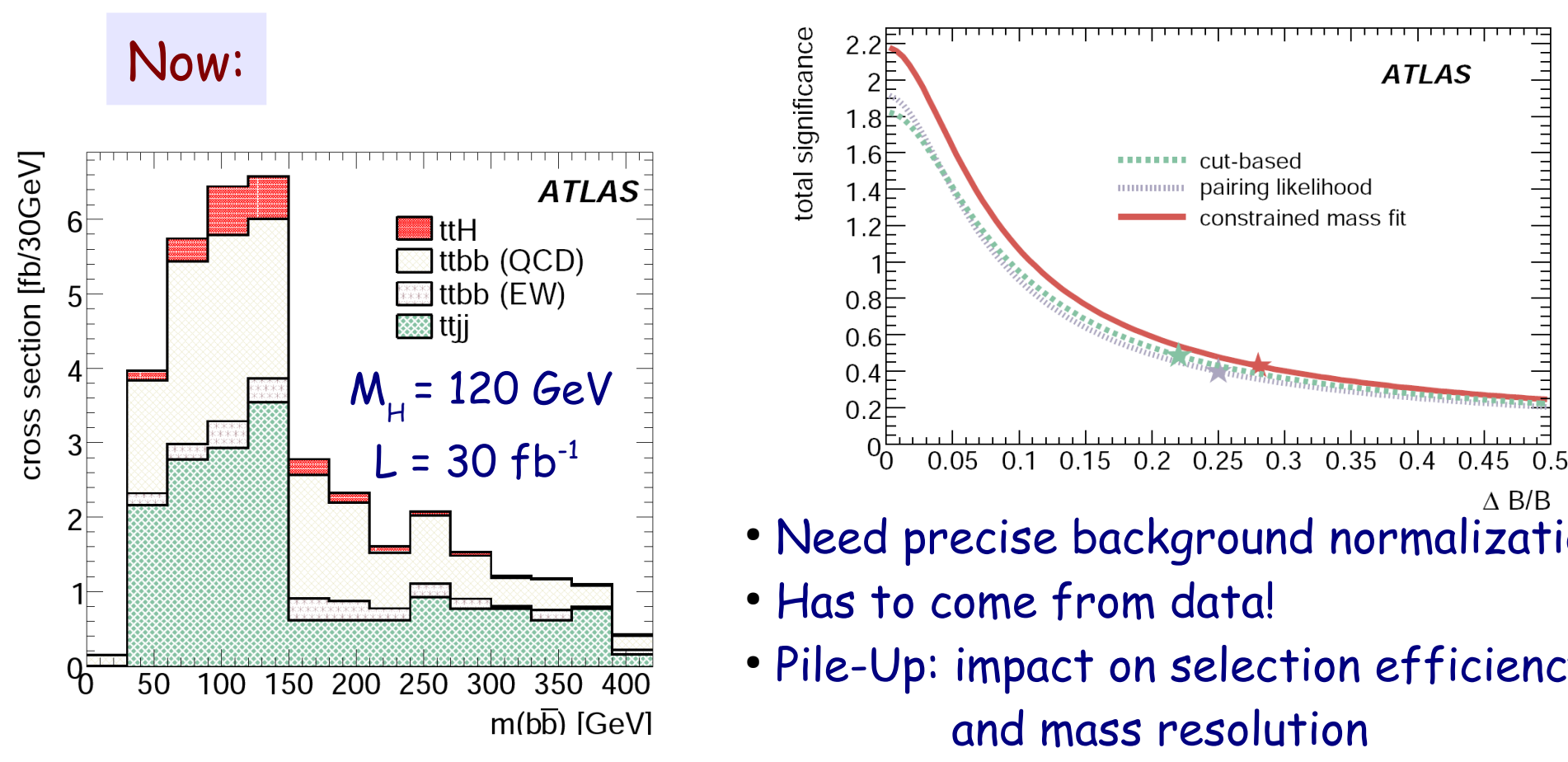

- Need precise background normalization!

- Has to come from data!

- Pile-Up: impact on selection efficiency and mass resolution

$\rightarrow \mathrm{ttH}$ has disappeared from latest sensitivity plots! 
The most important channels at the TEVATRON at low mass!

But: Signal to background ratio less favourable at the LHC
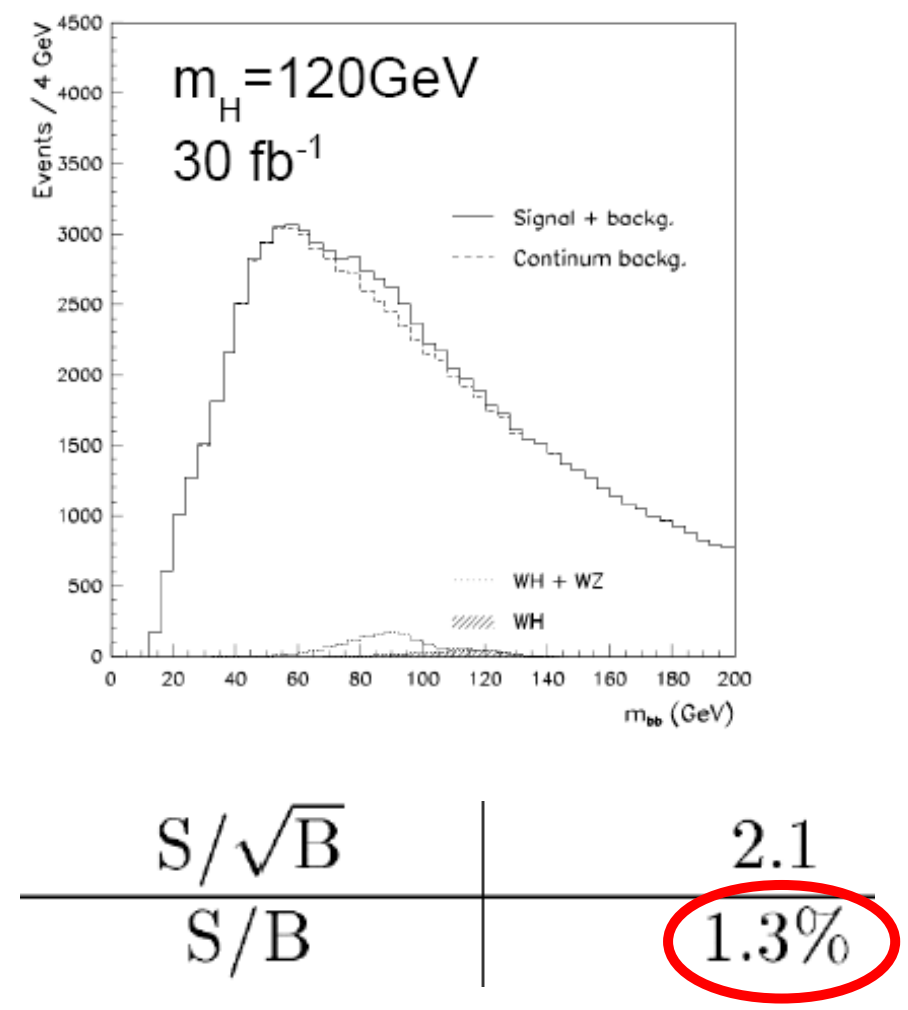

Follow idea of J.Butterworth et al. [PRL 100:242001,2008]:

Select events ( $\approx 5 \%$ of cross section), in which $H$ und $W$ bosons have large transverse momenta: $p_{T}>200 \mathrm{GeV}$

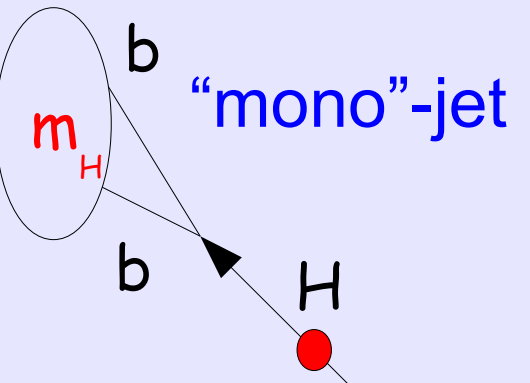

W4

$\rightarrow$ b-Quarks in one "fat" Jet

+ Acceptance (more central in detector)

+ Lepton-Identification, B-Tagging 


\section{W/ZH}

Analyse jet structure:
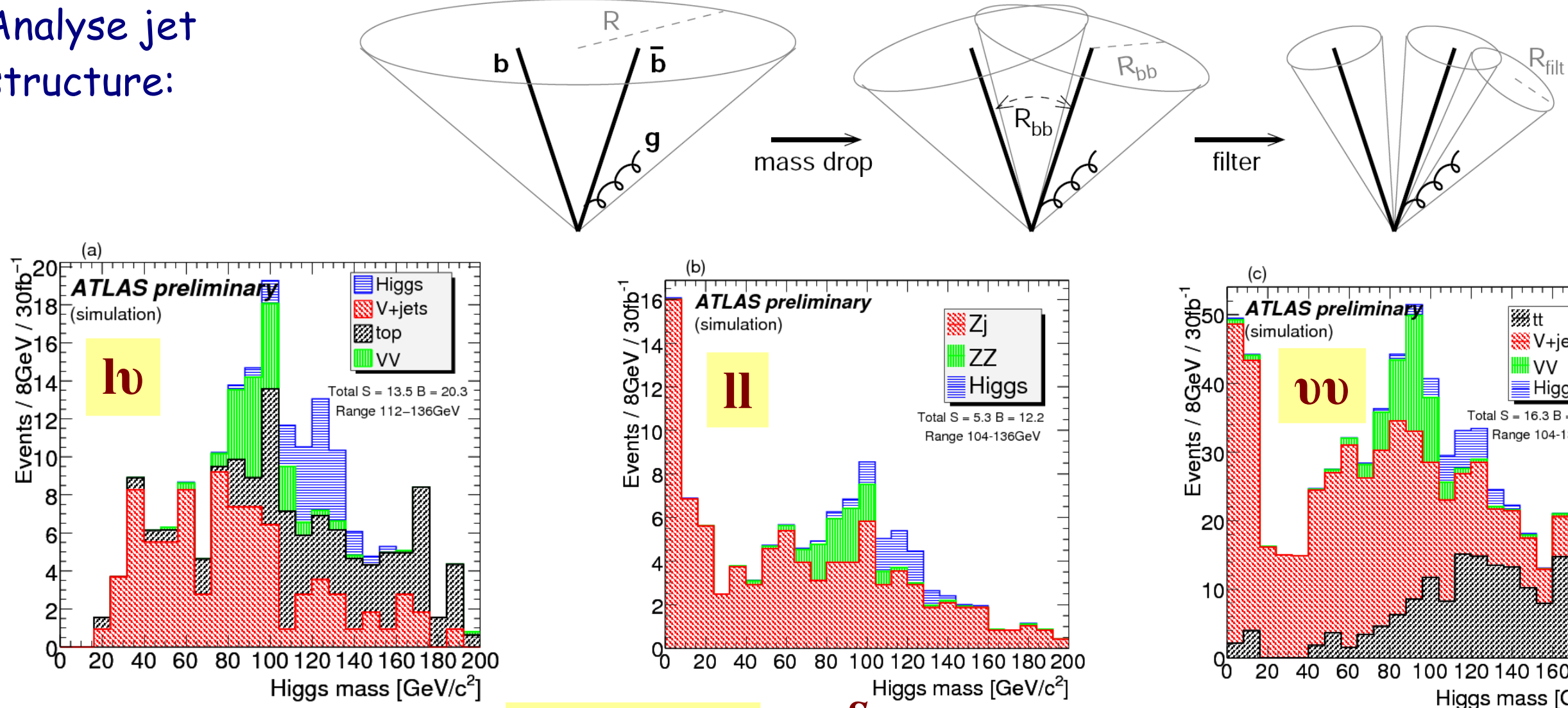

$$
L^{\text {int. }}=30 \mathrm{fb}^{-1}: \frac{S}{\sqrt{B}}=3.0
$$

Combined: $\frac{S}{\sqrt{B}}=\mathbf{3 . 7}$ (Pile-Up not yet included)

$$
M_{H}=120 \mathrm{GeV} \quad \frac{S}{\sqrt{B}}=1.5
$$

- S/B much better than for ttH

- Different backgrounds for different channels

- Still good sensitivity including systematics (e.g. $S / \sqrt{ } B=3.0$ for $15 \%$ uncertainty on all backgrounds) 
"Golden Channel":

- Clear, narrow mass peak on top of background

- Covers wide mass range

Backgrounds:

- Irreducible: ZZ

- Reducible: Zbb, ††

$\rightarrow$ Lepton isolation and impact parameters

Effect of Pile-Up $\left(10^{33} \mathrm{~cm}^{-2} \mathrm{~s}^{-1}\right)$ and Cavern Background:

Loss in signal efficiency of $\approx 10 \%$ (studied for $m_{H}=130 \mathrm{GeV}$ )
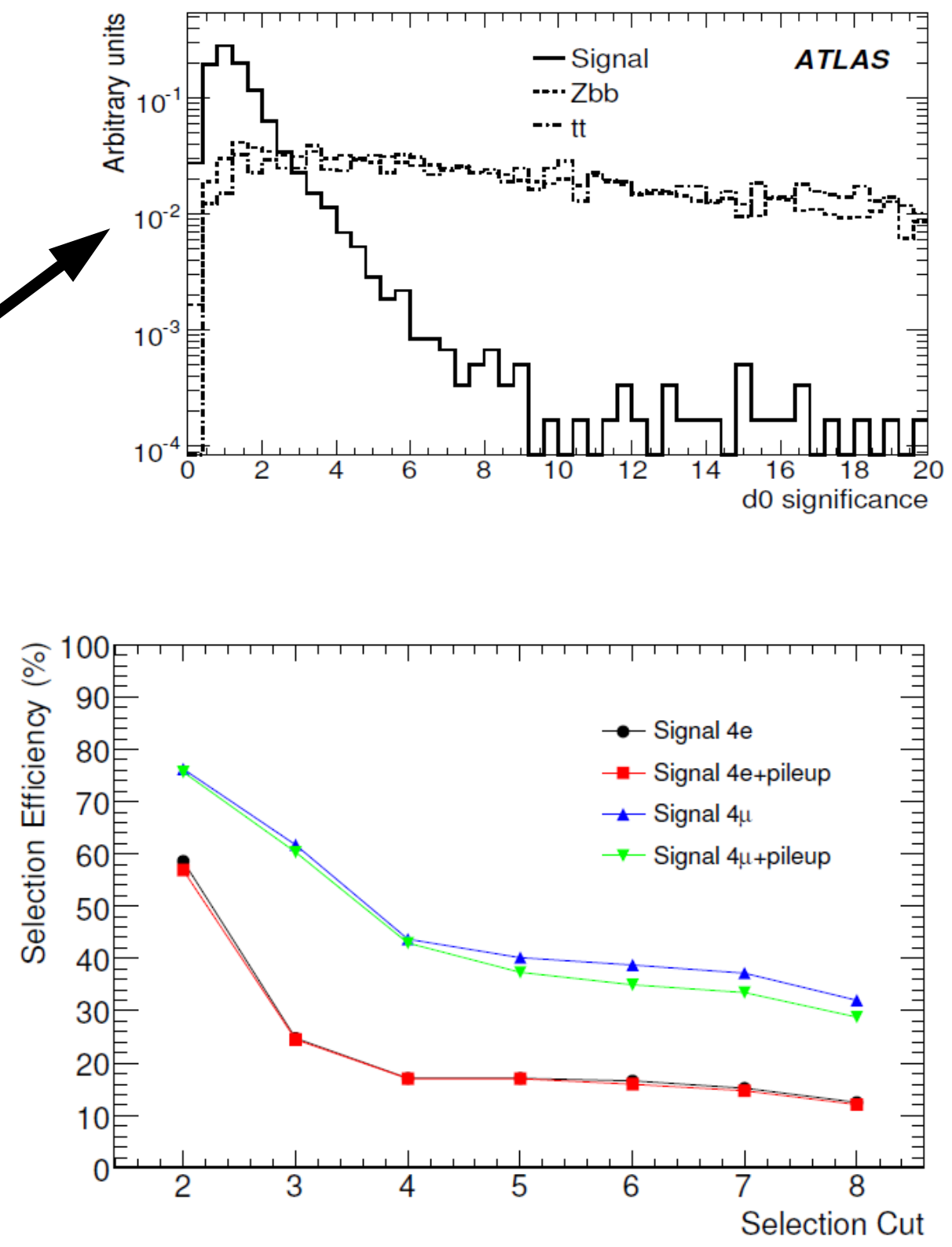


\section{$H \rightarrow Z Z$}
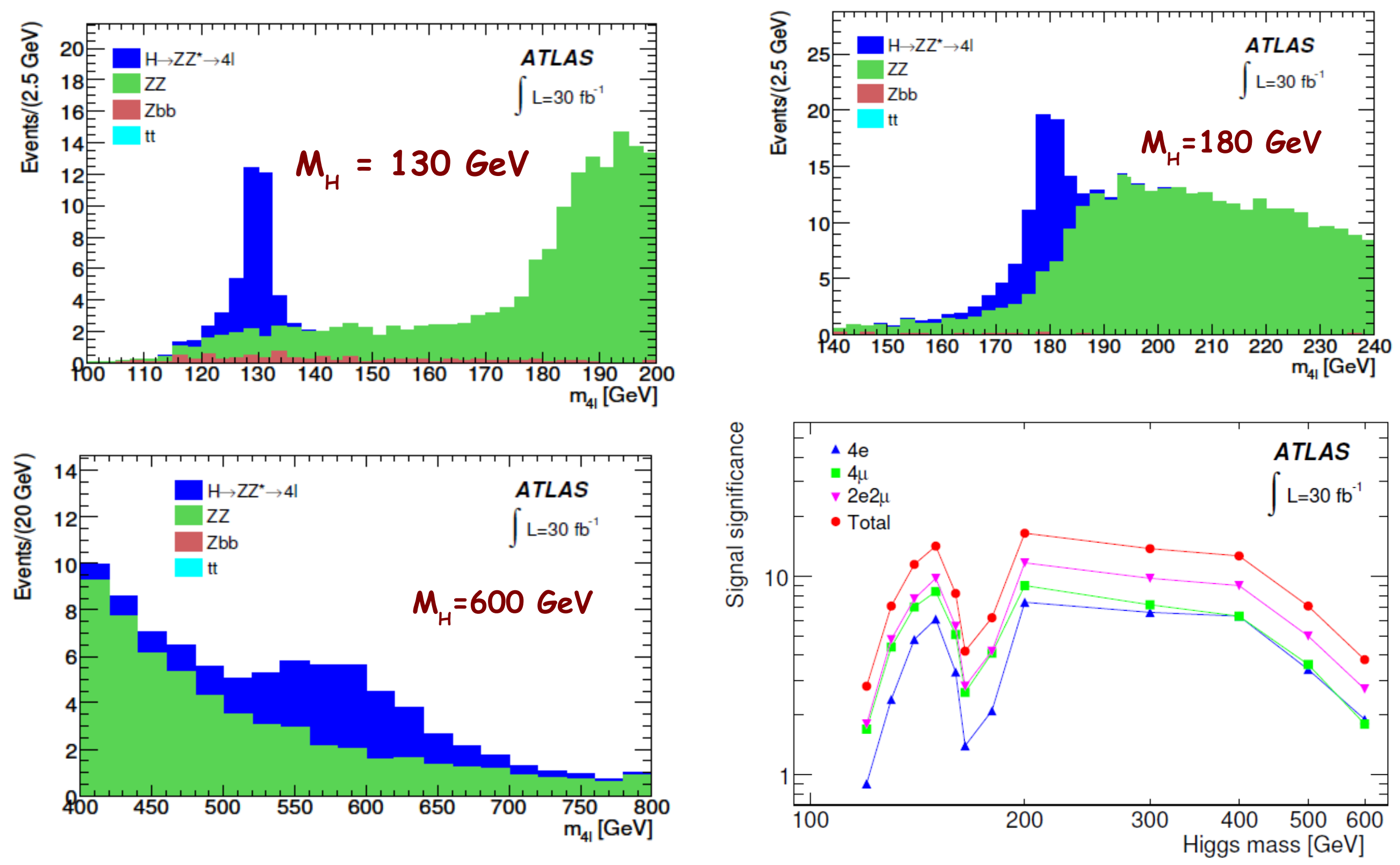


\section{$H \rightarrow W W$}

- Accessible for dominant production process (gg fusion) AND decay mode $\left(m_{H}>135 \mathrm{GeV}\right)$

- Final state considered: $\mathrm{H} \rightarrow \mathrm{WW} \rightarrow$ evpr

- Two analyses: + 0 jet (gg fusion), +2 jets (vector boson fusion)

- 2 neutrinos $\rightarrow$ no mass peak reconstructable, only transverse mass

- Use angular correlation between leptons:
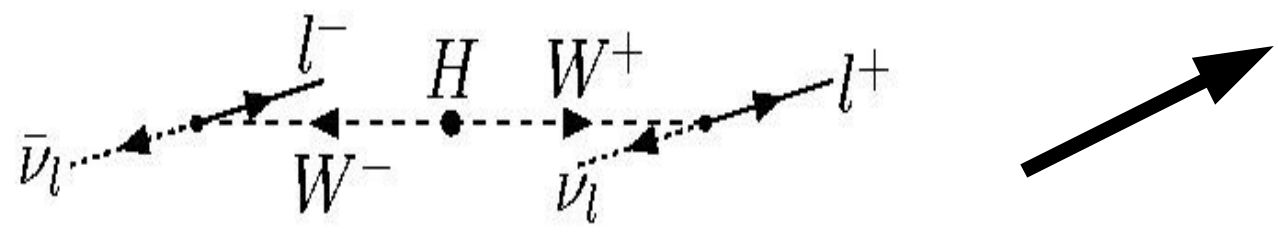

Fit transverse mass and transverse momentum of The WW system in $\Delta \Phi$ signal and control regions
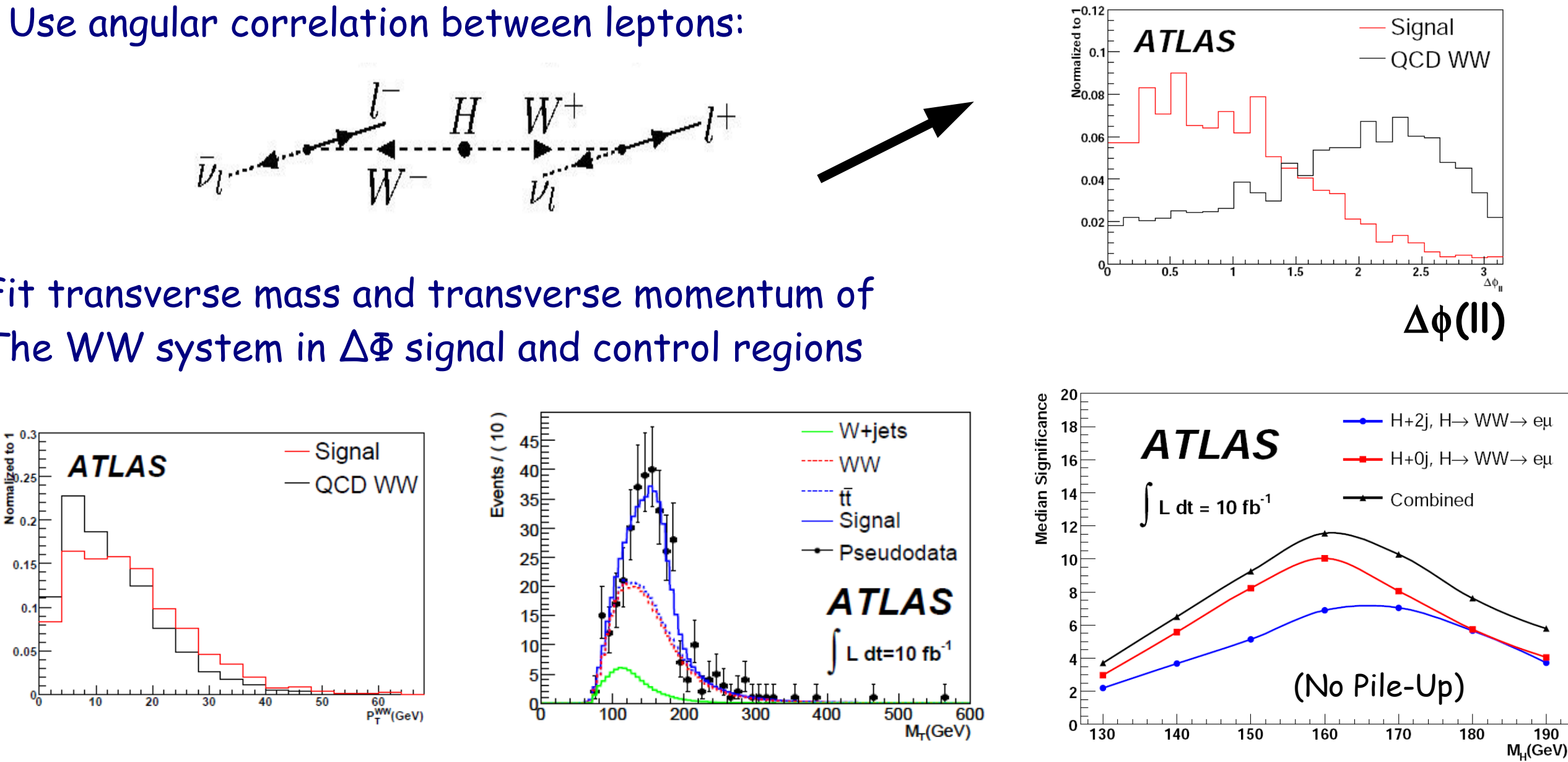


\section{Combined Discovery Potential}
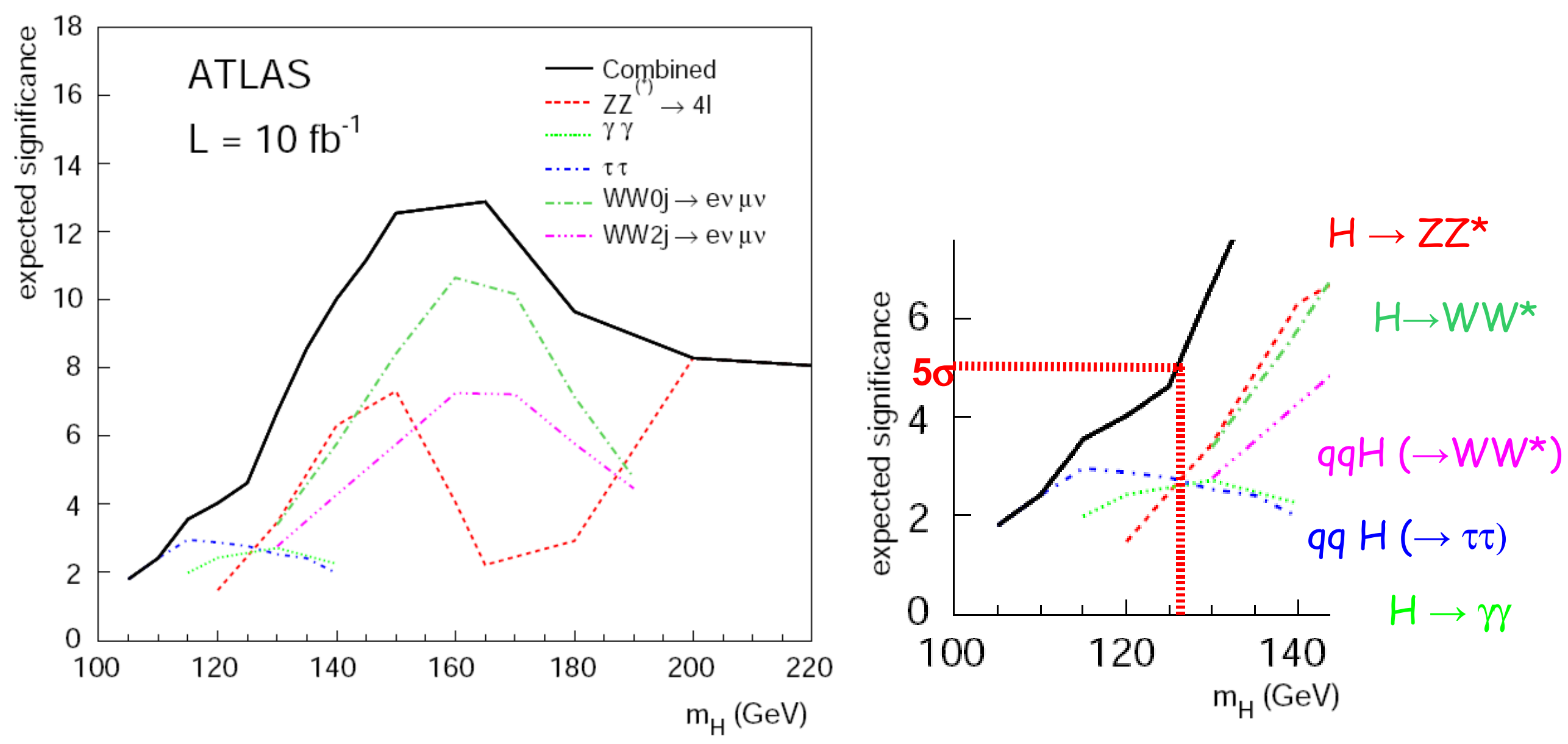

- $5 \sigma$ discovery seems possible with $10 \mathrm{fb}^{-1}$ for $m_{H}>127 \mathrm{GeV}$

- Adding the $\mathrm{W} / \mathrm{ZH}, \mathrm{H} \rightarrow$ bb channel will improve situation for low masses 


\section{Exclusion}

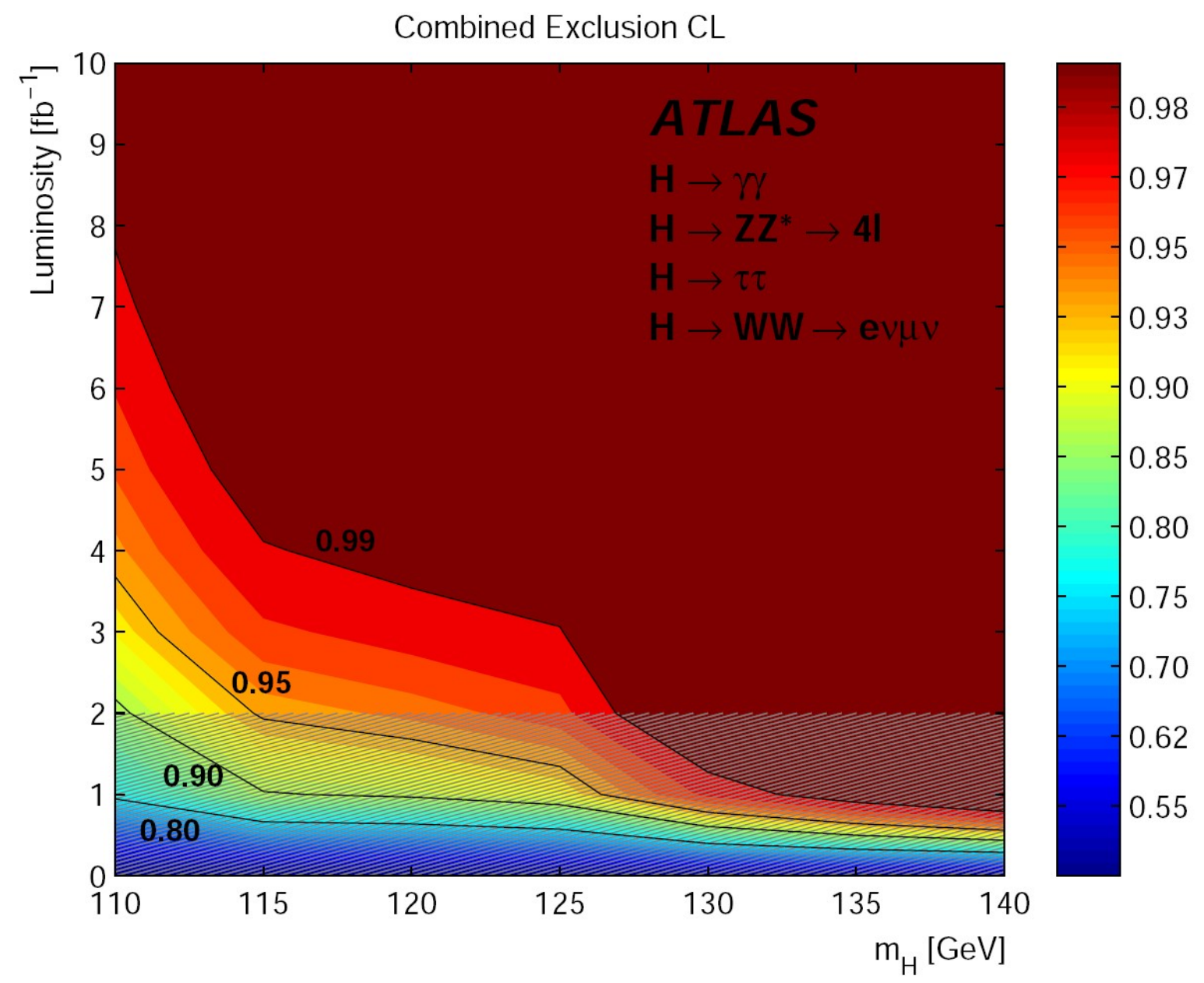

$2 \mathrm{fb}^{-1}$ to exclude $m_{H}>115 \mathrm{GeV} @ 95 \% \mathrm{CL}$ 


\section{Conclusions}

- Much more realistic detector simulations, Monte Carlo generators, reconstruction tools etc. have improved the quality of the predictions for ATLAS Higgs boson searches significantly

- Strong effort has been put in studying data driven methods to become less dependent on Monte Carlo generators

- A discovery (or exclusion) should be possible over the full mass range from $115 \mathrm{GeV}-1 \mathrm{TeV}$

- However, the low mass region (just above the LEP limit) is very challenging and several channels may contribute 